\title{
Epidermoid Cyst of the Cecum
}

\author{
Jae-Young Park, Youn Wha Kim, Kil Yeon Lee ${ }^{1}$, Ji-Youn Sung \\ Departments of Pathology and ${ }^{1}$ Surgery, Graduate School of Medicine, Kyung Hee University, Seoul, Korea
}

An epidermoid cyst of the cecum is extremely rare; only eight cases have been reported in the literature. A 63-year-old woman was admitted to Kyung Hee University Medical Center with a colonic mass that had been discovered incidentally during a regular health check-up. The radiographic impression was that this mass was a gastrointestinal stromal tumor. During surgery, an exophytic mass in the cecal wall was resected by using an ileocecectomy. Based on the macroscopic and the microscopic findings, this case was identified as an epidermoid cyst of the cecal wall. We report this case to discuss the origin of this entity and the unusual nature of our case.

\section{Keywords: Epidermoid cyst; Cecum}

\section{INTRODUCTION}

Epidermoid cysts rarely develop in the cecum, yet they have been reported in other internal organs, including the testes, spleen, accessory spleen, liver and kidneys [1]. Only eight cases of cecal epidermoid cysts have been reported in the English literature [1-8]. Epidermoid cysts are generally considered to be sequestration cysts that may be congenital or acquired. An acquired epidermoid cyst develops in a patient with a history of abdominal trauma or surgery whereas a congenital epidermoid cyst may be the result of an aberrant embryogenic ectodermal implantation during embryogenesis. We report the case of a cecal epidermoid cyst in an elderly woman with no history of abdominal surgery or trauma. The cyst was discovered incidentally and was resected by using an ileocecectomy.

\section{CASE REPORT}

A 63-year-old female was admitted to Kyung Hee University Medical Center after an incidental finding of a right colonic mass during her regular health check-up. She underwent surgical excision

Received: April 8, 2014 - Accepted: May 21, 2014

Correspondence to: Ji-Youn Sung, M.D.

Department of Pathology, Kyung Hee University Medical Center,

23 Kyungheedae-ro, Dongdaemun-gu, Seoul 130-872, Korea

Tel: +82-2-958-8734, Fax: +82-2-958-8730

E-mail: jyune15@gmail.com

(C) 2015 The Korean Society of Coloproctology

This is an open-access article distributed under the terms of the Creative Commons Attribution NonCommercial License (http://creativecommons.org/licenses/by-nc/3.0) which permits unrestricted noncommercial use, distribution, and reproduction in any medium, provided the original work is properly cited. of a submandibular gland due to a Wharton's duct stone. She was asymptomatic. On physical examination, there were no signs of fever or acute abdomen and no palpable abdominal mass. Colonoscopy revealed a mucosal tumor in the cecum without mucosal abnormalities. On abdominal computed tomography (CT), there was a 3.8-cm exophytic mass with internal low attenuation between the cecum and the terminal ileum (Fig. 1). The radiographic impression was that this mass was a gastrointestinal stromal tumor (GIST) and likely was not an adenocarcinoma. On further exploration, a round, soft, exophytic mass was identified at the cecal wall, $2.0 \mathrm{~cm}$ from the appendix orifice. After surgical investigation of the mass, an ileocecectomy was performed.

Macroscopically, the surface of the mass revealed an irregular cystic lesion filled with a yellowish-gray, cheesy material (Fig. 2). No connection between the cyst and the cecal lumen was observed. The mucosal and serosal surfaces of the cecum were unremarkable. Histologically, the wall of the cyst was surrounded in its entirety by a cecal muscularis propria. Cells lining the cyst formed a mature, keratinized, and stratified squamous epithelium with a granular layer (Fig. 3A). The focal area of cystic wall showed nonkeratinizing squamous epithelium without a granular layer (Fig. 3B). The lumen of the cyst was filled with mature, dense keratin. Skin adnexa were completely absent. No other ectodermal, endodermal or mesenchymal elements were noted. The pathological diagnosis was an epidermoid cyst.

\section{DISCUSSION}

Epidermoid cysts are generally believed to be sequestration cysts that may be congenital or acquired. Congenital epidermoid cysts 


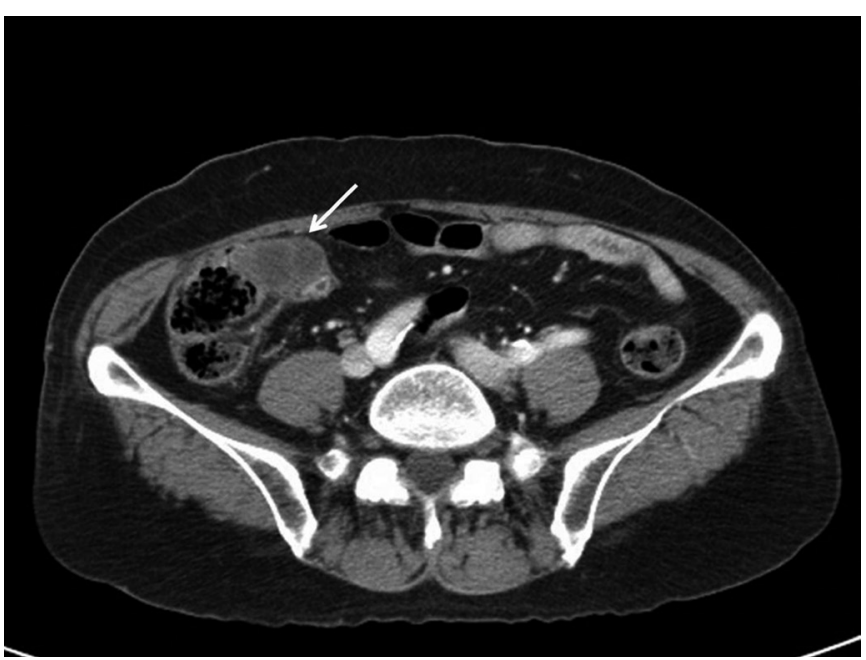

Fig. 1. Abdominal computed tomography revealed an exophytic mass with internal low attenuation between the cecum and the terminal ileum (arrow).

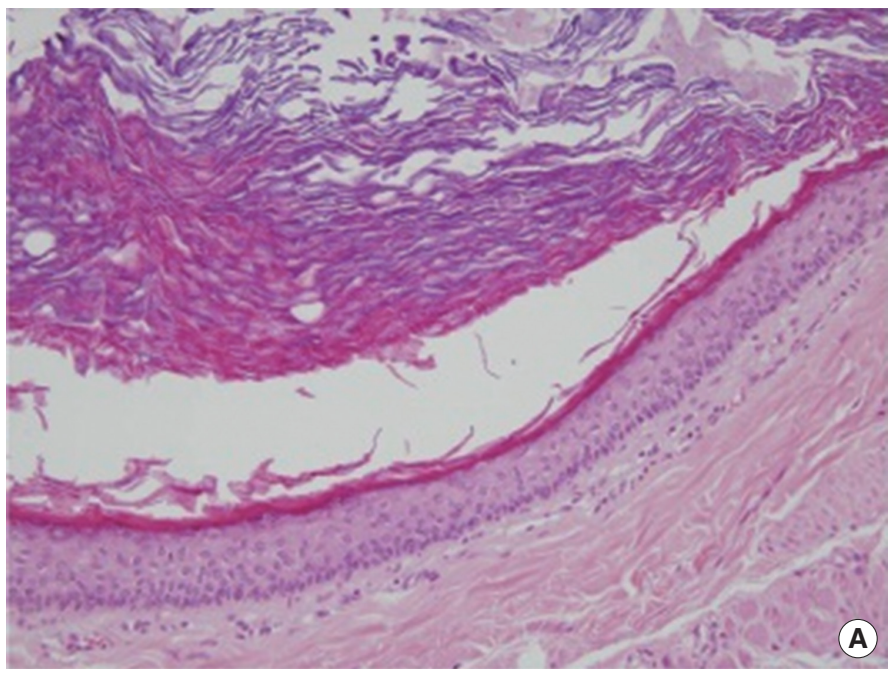

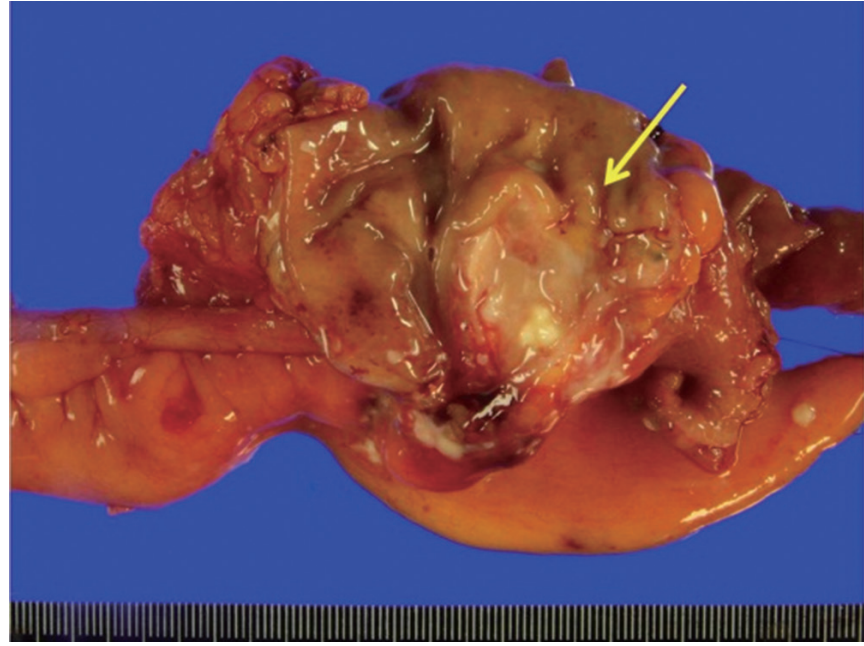

Fig. 2. Gross photograph of the ileocecectomy specimen showing the intramural cystic lesion filled with a yellow-to-gray cheesy material (arrow)

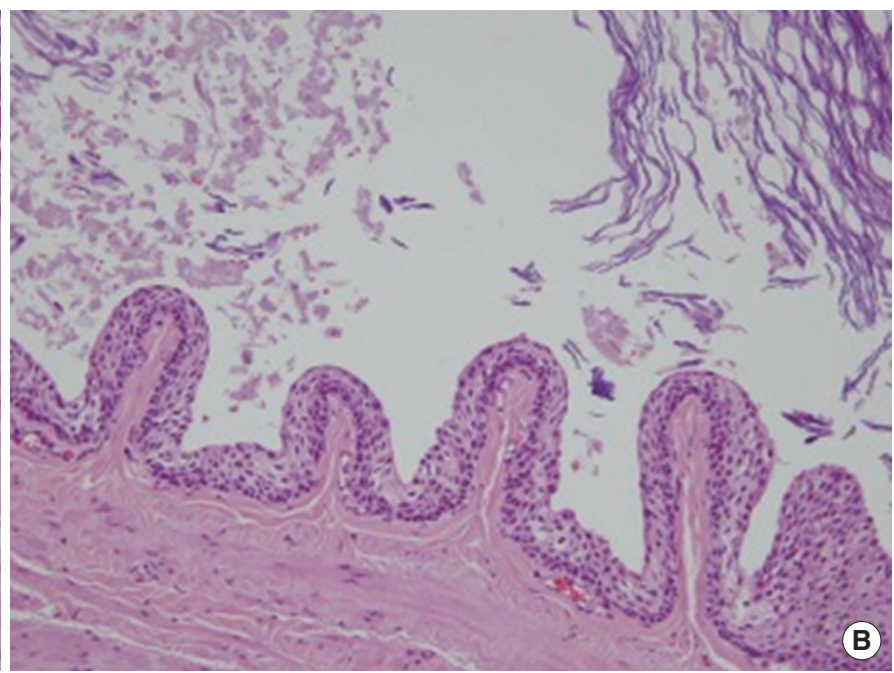

Fig. 3. (A) The cyst is lined by a keratinizing, stratified, squamous epithelium with a granular layer $(\mathrm{H} \& \mathrm{E}, \times 100)$. (B) The focal area of the cyst has nonkeratinizing, squamous, epithelial-lining cells $(\mathrm{H} \& \mathrm{E}, \times 100)$.

are related to implantation of ectodermal elements at the time of closure of the neural groove or to the coalescence of other epithelial fusion lines, which typically occur in the head, neck, and anorectal areas and frequently involve the central nervous system and the intraspinal regions [9]. Epidermoid cysts can occur in the testes, spleen, accessory spleen, liver, and kidneys [1]. Acquired epidermoid cysts are traumatic or iatrogenic and may be caused by the implantation of the epidermis in locations favorable for growth [4].

Epidermoid cysts of the cecum are extremely rare, with only eight reported cecal epidermoid cysts in the English literature (Table 1) [1-8]. Three of the reported cecal epidermoid cyst cases were as- sociated with a history of abdominal surgery. Two had undergone an appendectomy 12 years and 16 years prior to the diagnosis of a cecal epidermoid cyst $[2,4]$. One patient had had a cesarean section [7]. These cases were attributed to iatrogenic implantation of epidermal fragments via a scalpel, needle or clamp during the operation $[2,4,7]$. The remaining patients had no history of abdominal surgery, and their cecal epidermoid cysts were considered to be congenital lesions. Andiran et al. [5] suggested that an inclusion or closure line of an epidermal structure may occur when the cecum re-enters the abdominal cavity during intrauterine rotation in the final steps of gut development. This encasing of an epidermal structure may result in later development of a cecal epider- 
Table 1. Reported cases of cecal epidermoid cysts

\begin{tabular}{|c|c|c|c|c|c|c|c|}
\hline Case No. & Age (yr) & Sex & History of operation & Initial diagnosis & $\begin{array}{l}\text { Location of } \\
\text { epidermoid cyst }\end{array}$ & Year reported & Reference \\
\hline 1 & 53 & Male & Appendectomy & Right lower abdominal mass & Intramural & 1961 & 2 \\
\hline 3 & 71 & Male & Appendectomy & Intramural cecal mass & Intramural & 1969 & 4 \\
\hline 4 & 8 & Female & None & Right lower abdominal cyst & Subserosal & 1999 & 5 \\
\hline 7 & 31 & Female & Cesarean surgery & Adnexal mass & Subserosal & 2012 & 7 \\
\hline 8 & 54 & Male & None & Mesenteric cyst & Subserosal & 2013 & 8 \\
\hline 9 & 63 & Female & None & GIST & Intramural & Our case & \\
\hline
\end{tabular}

GIST, gastrointestinal stromal tumor.

moid cyst. Our patient had no history of abdominal surgery; therefore, her epidermoid cyst may have occurred due to an aberrant, embryonic, ectodermal implantation during embryogenesis.

The differential diagnosis of an epidermoid cyst is rarely considered in patients with cecal cystic lesions. Because epidermoid cysts of the cecum are mostly located in the subserosal area $[1,3,5-8]$, On CT imaging, these cysts may be confused with other intra-abdominal cystic lesions, including mesenteric cysts, lymphatic cysts, appendiceal mucoceles or duplication cysts $[1,8]$. Especially, in female patients, they may initially be diagnosed as a right adnexal mass or cyst, so the differential diagnosis of an ovarian cyst or a cecal epidermoid cyst must be considered [3, 7]. Interestingly, our patient had an initial diagnosis of a GIST based on CT findings. This initial diagnosis was likely based on the unusual intramuscular location of the epidermoid cyst. In a previous case of a 71-yearold male patient, an epidermoid cyst within the cecal wall was considered to be an intramural cecal mass based on radiologic findings; the mass was then resected locally [4]. Grossly and histologically, epidermoid cysts resemble dermoid cysts. Dermoid cysts are lined with mature, squamous, epithelial cells and specialized skin structures, such as hair follicles, sweat glands and sebaceous glands. However, epidermoid cysts lack specialized skin structures, which differentiates them from dermoid cysts [9].

In conclusion, the development of an epidermoid cyst in the cecum, especially in the intramuscular region, is a rare occurrence; thus, epidermoid cysts are very rarely included in the differential diagnosis of cecal masses. From this case, we found that, given their location on radiographic imaging, cecal epidermoid cysts can be confused with mesenchymal cecal masses such as GISTs. Therefore, the possibility of an epidermoid cyst should be considered in the differential diagnosis for submucosal or intramuscular masses in the cecal area.

\section{CONFLICT OF INTEREST}

No potential conflict of interest relevant to this article was reported.

\section{REFERENCES}

1. Uzunlar A, Bukte Y. Epidermoid cyst of the cecum in an elderly man. Ann Saudi Med 2006;26:477-9.

2. Pan A, Rogers AG, Klass AA. Epidermoid cyst of the cecum. Can Med Assoc J 1961;84:1075.

3. Chanddreviotis N. Epidermoid cyst of the cecum. JAMA 1965; 192:425-6.

4. Pear BL, Wolff JN. Epidermoid cyst of the cecum. JAMA 1969;207: 1516-7.

5. Andiran F, Dayi S, Caydere M, Dilmen G, Dilmen U. Epidermoid cyst of the cecum. J Pediatr Surg 1999;34:1567-9.

6. Mady HH, Melhem MF. Epidermoid cyst of the cecum of an elderly man with no previous history of surgery: a case report and review of literature. Int J Colorectal Dis 2002;17:280-3.

7. Demır H, Aydogan B, Sahın H, Ocal P, Ilvan S. Epidermoid cyst of the cecum: a case report. Turk J Gastroenterol 2012;23:406-9.

8. Sahoo MR, Gowda MS, Behera SS. Unusual site and uncommon presentation of epidermoid cyst: a rare case report and review of literature. BMJ Case Rep 2013;2013:bcr2012007907.

9. Pear BL. Epidermoid and dermoid sequestration cysts. Am J Roentgenol Radium Ther Nucl Med 1970;110:148-55. 